$B^{2} \div 0$ 4. 4 :
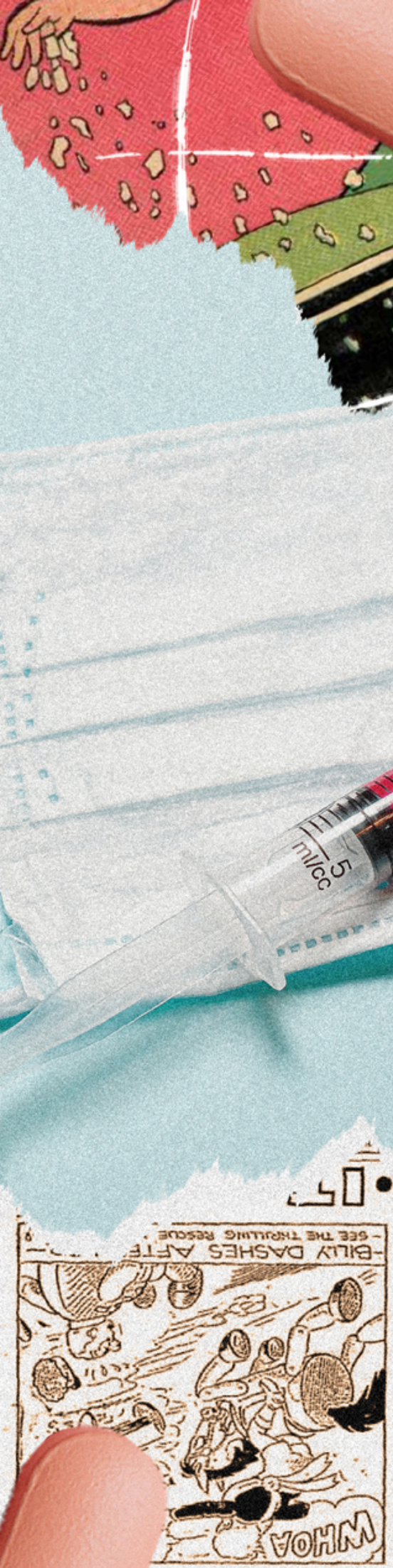

\title{
- $5[0$
}

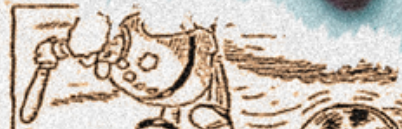

逐

8. 8
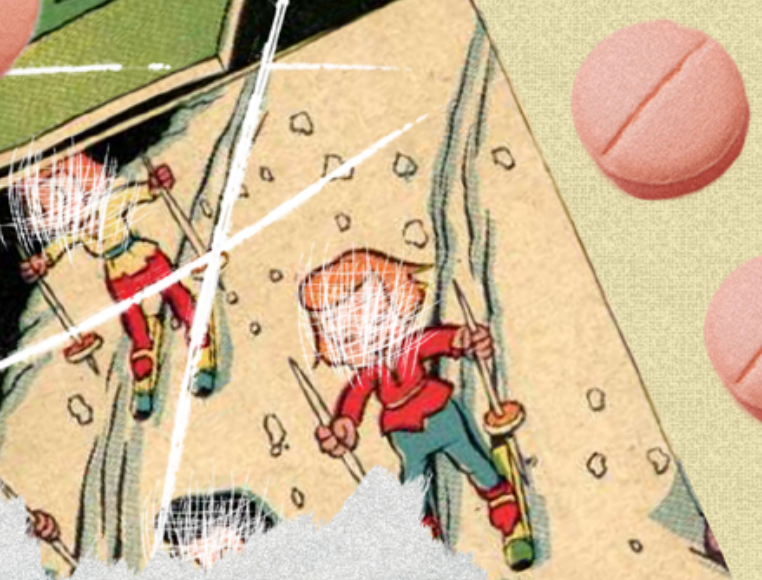

i。

त्वेजए।
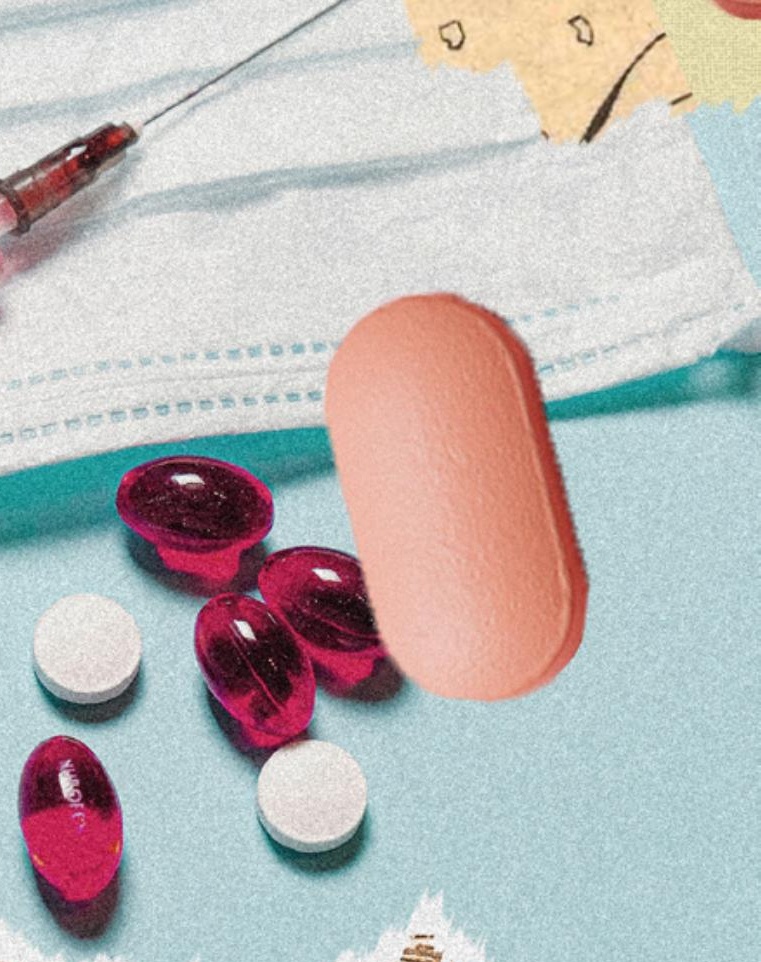

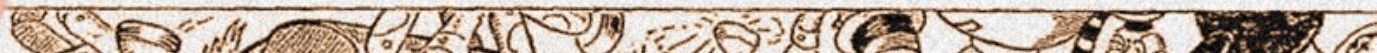




\section{Histórias em quadrinhos para a popularização do uso racional de medicamentos}

\section{Comics to popularize the rational use of medications}

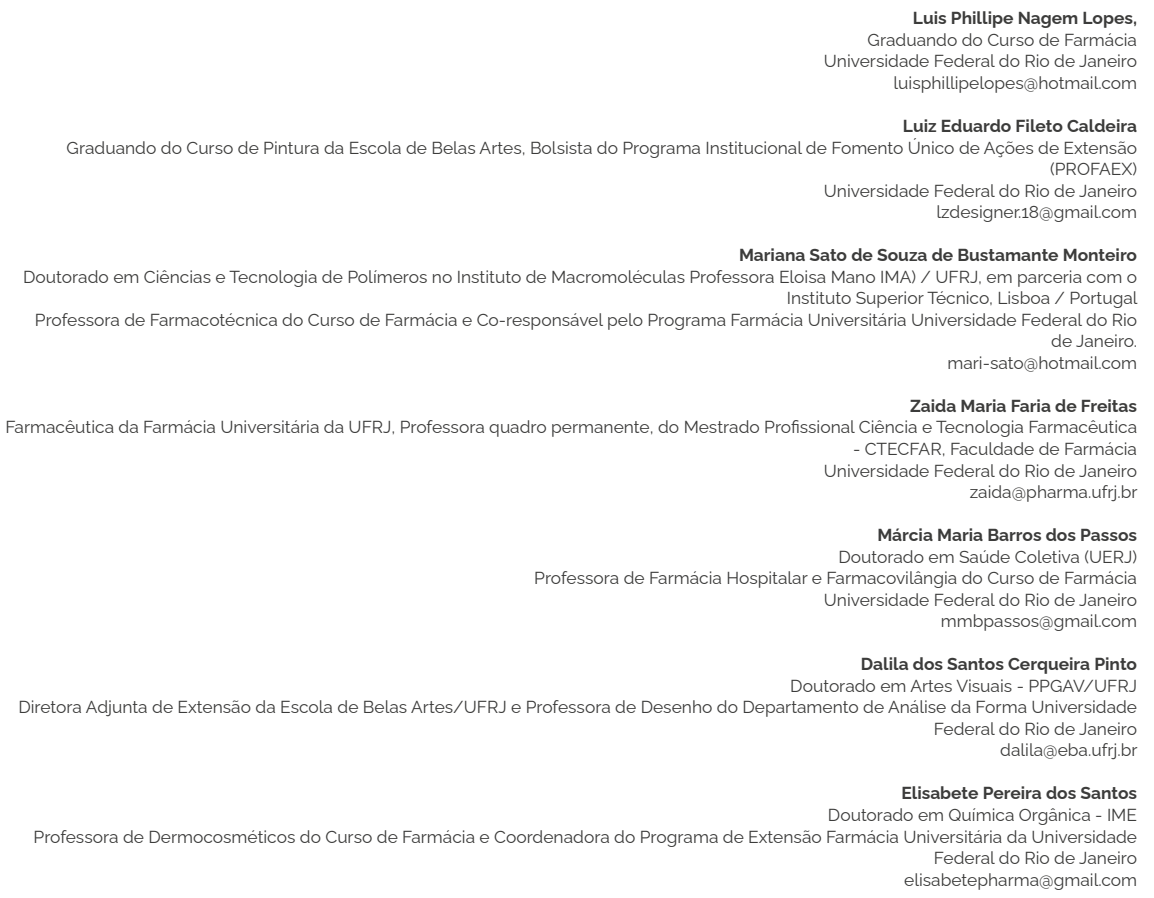

\section{RESUMO}

O uso irracional de medicamentos é uma preocupação mundial, por isso a educação em saúde é fundamental para mitigação dos riscos associados a essa prática. O presente trabalho tem por objetivo descrever as atividades de promoção do uso racional de medicamentos desenvolvidos pela Farmácia Universitária na Semana Nacional de Ciência e Tecnologia (SNCT). Trata-se de um relato de experiência sobre a elaboração de histórias em quadrinhos e o desenvolvimento de atividades com cerca de cinco mil alunos do ensino médio e fundamental de escolas públicas, localizadas no municipio do Rio de Janeiro. Os temas abordados como caminho para ampliar o conhecimento dos participantes foram: o uso racional de antimicrobianos; automedicação; medicamentos fora do prazo de validade: descarte de medicamentos e descontinuidade no tratamento. Pode-se concluir que as histórias em quadrinhos foram instrumentos eficientes para educação em saúde e também uma estratégia para o seu desenvolvimento na SNCT de 2019

Palavras-chave: Uso racional de medicamentos. Educação em saúde. Popularização do conhecimento. Histórias em quadrinhos.

\section{ABSTRACT}

The irrational use of medicines is a worldwide concern, so health education is essential to mitigate this practice in health. The present work aimed to describe the activities to promote the rational use of medicines developed by the University Pharmacy (FU), in the National Week of Science and Technology (SNCT). It is an experience report describing the elaboration of comic books and the development of activities with about five thousand students from high school and elementary schools in public schools, located in the city of Rio de Janeiro. The topics covered: the rational use of antimicrobials; self-medication; medicines out of date; disposal of drugs and discontinuity in treatment, as a way to increase the knowledge of the participants. It can be concluded that comics were efficient instruments for health education and a strategy for their development in SNCT 2019.

Keywords: Rational Use of Medicines. Health Education. Popularization of Knowledge. Stories in Comics. 


\section{INTRODUÇÃO}

O Uso Racional de Medicamentos (URM) - definido pela Organização Mundial de Saúde (OMS) como "quando pacientes recebem medicamentos apropriados para suas condições clínicas, em doses adequadas às suas necessidades individuais, por um período adequado e ao menor custo para si e para a comunidade" (WHO, 1985) -, vem sendo uma problemática recorrente, já que em todo o mundo mais de $50 \%$ de todos os medicamentos são receitados, dispensados e vendidos de forma inadequada (WHO, 2015), configurando-se o uso irracional de medicamentos, isto é, a utilização fora do contexto de URM.

No Brasil, o uso irracional ou inadequado dos medicamentos é influenciado por questões como a automedicação (Arrais et al., 1997), propaganda e publicidade tendenciosas (Luchessi et al., 2005), que como consequência podem ocasionar intoxicações e até mesmo mortes (Matos et al., 2002), permeando, assim, todos os elos inerentes ao ciclo da assistência farmacêutica - seleção, programação, aquisição, armazenamento, distribuição e dispensação -e da indústria farmacêutica, que tem fomentado a criação de novas necessidades de consumo de medicamentos pela publicidade tendenciosa e formado uma sociedade "medicalizada" (Barros, 2004).

Uma pesquisa recente investigou o uso inadequado de medicamentos pela população urbana brasileira e verificou que 46,1\% das pessoas apresentaram pelo menos um comportamento de uso inadequado de medicamentos (Luiza et al., 2019). Segundo os autores, a alta prevalência de uso inadequado de medicamentos, associado a características individuais do paciente e ao sistema de saúde, indicam a necessidade urgente de estabelecer prioridades quanto à educação em saúde e intervenções públicas que mitiguem o uso irracional de medicamentos.

A educação em saúde - que é estimulada internacionalmente - é uma estratégia capaz de gerar ferramentas para a redução do uso irracional de medicamentos e amparar os usuários acerca das mudanças de comportamentos e atitudes necessárias para melhorias nas condições de saúde (OPAS, 2007). As ações de educação em saúde têm se mostrado benéficas em vários paises no contexto escolar, mas também em comunidades (Darnaud \& Dato, 2009; Oliveira \& Presoto, 2009). Nesse sentido, tais ações necessitam de ferramentas que transmitam de forma significativa o ensino. Por isso, várias técnicas de aprendizado sobre o uso de medicamentos são utilizadas: videotapes, circuitos práticos, técnicas de dramatização, cartazes, uso de fantoches, etc. As histórias em quadrinhos também vêm sendo utilizadas como estratégia de ensino para aprendizado em ciência e informações sobre saúde, principalmente para o público infantil (Negrete, 2019; Rebolho et al., 2009).

No contexto da educação em saúde, a extensão é o instrumento necessário para que os produtos da Universidade, a pesquisa e o ensino, estejam articulados entre si, e possa promover a interação transformadora com os outros setores da sociedade por meio de uma interação dialógica, com troca 
de conhecimentos e contato com as questões contemporâneas presentes no contexto social. Nos projetos de extensão, cuja finalidade é a melhoria na qualidade de vida da população, a extensão tem um papel de responsabilidade social e faz parte de uma nova cultura, que provoca a maior e a mais importante mudança registrada no ambiente acadêmico e corporativo nos últimos anos (Carbonari \& Pereira et al., 2015).

Portanto, a extensão tem papel essencial tanto na vida dos acadêmicos, que colocam em prática tudo o que aprendem em sala de aula, quanto na vida das pessoas que usufruem desse aprendizado. A Extensão Universitária, nesse contexto, é um importante instrumento capaz de propagar o conhecimento científico de forma lúdica, educativa e interessante (Rodrigues et al., 2013).

As histórias em quadrinhos podem ser utilizadas como um instrumento de ensino e motivação para o aprendizado de conceitos relacionados a novos hábitos em saúde, já que elas podem transmitir conhecimentos científicos de forma atrativa e divertida, facilitando a memorização desses conceitos (Negrete \& Lartigue, 2004). Ademais, as histórias em quadrinhos estão inseridas no currículo escolar em vários países (Rama \& Vergueiro, 2008); nas práticas educativas, elas podem favorecer a comunicação de um conhecimento especifico. Vale ressaltar que as histórias em quadrinhos não atraem somente crianças e adolescentes, elas são difundidas também entre jovens e adultos, servindo para exemplificar e informar sobre problemas enfrentados pela população contemporânea, dentre os quais a temática da saúde deve estar presente (Alcântara \& Bezerra, 2016).

Sabendo-se que, no Brasil, ocorre o uso indiscriminado de medicamentos e que tal uso acarreta uma série de problemas para a saúde (Brasil, 2012), e também sabendo que é cada vez mais frequente e prematura a utilização indiscriminada de medicamentos pela população, educar e informar a população acerca das consequências do uso não racional de medicamentos é essencial para uma mudança de hábito em relação aos medicamentos. Em concordância com o exposto, esse estudo tem como objetivo relatar a experiência do desenvolvimento de uma história em quadrinhos utilizada como recurso didático para orientar e incentivar o Uso Racional de Medicamentos (URM) na Semana Nacional de Ciência e Tecnologia (SNCT), 2019. A SNCT tem como principal objetivo aproximar a ciência e a tecnologia da população, promovendo atividades de divulgação cientíica em todo o país. Seu principal objetivo é criar uma linguagem acessivel à população, principalmente no ambiente escolar - já que crianças e jovens são considerados o principal público do evento - e utilizar meios inovadores para estimular a curiosidade e motivar a discussão sobre as implicações sociais da ciência, além de aprofundar conhecimentos sobre determinado tema (Bonfim, 2015). 


\section{MÉTODO}

Utilizou-se a estratégia qualitativa, baseada no relato de experiência, para descrever o processo de desenvolvimento da atividade educativa, na qual os pesquisadores e participantes representativos da situação estavam envolvidos de modo cooperativo ou participativo (Minayo, 2008).

Os sujeitos deste estudo foram todos os participantes da SNCT 2019. A edição de 2019 abordou o tema "Bioeconomia: diversidade e riqueza para o desenvolvimento sustentável" e alcançou um público estimado de cinco mil estudantes do ensino médio e fundamental das escolas da rede pública, localizadas na cidade do Rio de Janeiro, além dos alunos e professores da Farmácia Universitária da UFRJ (FU-UFRJ).

\section{ETAPAS DO PLANEJAMENTO}

Os alunos da FU-UFRJ, junto com farmacêuticos e docentes da FU-UFRJ, selecionaram tópicos relativos ao URM, com base no que é fundamentado na literatura acerca dos principais problemas encontrados em relação aos medicamentos. O conceito norteador foi a definição preconizada pela OMS sobre URM. Assim, definiu-se que a história em quadrinhos abordaria os seguintes tópicos dentro do escopo de URM:

a) Acompanhamento multiprofissional do uso de antibióticos;

b) Automedicação de medicamentos isentos de prescrição (MIPS);

c) Uso de medicamentos vencidos;

d) Descontinuidade do tratamento por questões relacionadas ao acesso;

e) Descarte incorreto de medicamentos.

A estratégia educativa levou em consideração a diversidade do público que estaria na SNCT. Assim, optou-se pela história em quadrinhos, pois, além de atrair o público infantil, também abrange jovens e adultos (Alcântara \& Bezerra, 2016). Ademais, a história em quadrinhos realiza a integração entre a linguagem escrita e visual, atuando na construção de conhecimentos, na atribuição de sentidos e na formação de competências artísticas, literárias e linguísticas. Caruso e colaboradores (2002) enfatizam a utilização de tirinhas e de histórias em quadrinhos para a disseminação do conhecimento. Segundo estes autores, as tirinhas têm grande poder de concisão, já que ressaltam a linguagem da imagem objetivando a aprendizagem e despertando a curiosidade, permitindo a reflexão e o aprendizado por meio das próprias deduções e conclusões dos leitores. 


\section{ELABORAÇÃO DA HISTÓRIA EM QUADRINHOS}

O referencial teórico, bem como os tópicos levantados pelos alunos da FU-UFRJ, oriundos da literatura, foram transferidos para os alunos de Pintura da Escola de Belas Artes da UFRJ (EBA-UFRJ) a fim de elaborarem o conteúdo gráfico da história em quadrinhos. Visando uma interação dialógica efetiva, os alunos da FU-UFRJ e da EBA-UFRJ, dentro de suas competências, tiveram encontros quinzenais para discutir a adequação da linguagem e as estratégias para atrair a o público da SNCT, tendo em vista sua diversidade. Tais questões fomentam a ideia de que cada área do conhecimento e cada público-alvo possuem diferentes exigências que devem ser levadas em conta durante a produção das ilustrações (Morato et al., 1998).

\section{A AÇÃO NA SNCT}

A SNCT foi estabelecida pelo Decreto de 9 de junho de 2004. Ela é realizada sempre no mês de outubro sob a coordenação do Ministério da Ciência, Tecnologia, Inovações e Comunicações, por meio do Departamento de Difusão e Popularização da Ciência e Tecnologia (DEPDI/SECIS), e conta com a colaboração de secretarias estaduais e municipais, agências de fomento, espaços científico-culturais, instituições de ensino e pesquisa, sociedades científicas, escolas, órgãos governamentais, empresas de base tecnológica e entidades da sociedade civil. A semana tem o objetivo de aproximar a ciência e tecnologia da população, promovendo eventos que congreguem centenas de instituições a fim de realizarem atividades de divulgação científica em todo o país. A ideia é criar uma linguagem acessivel à população, por meios inovadores que estimulem a curiosidade e motivem a população a discutir as implicações sociais da ciência, além de aprofundarem seus conhecimentos sobre o tema (Universidade Federal do Rio de Janeiro, 2004).

Durante a SNCT, a UFRJ realiza exposições de ciência e tecnologia nos campos Cidade Universitária, Polo Caxias e Polo Macaé. O ginásio da Escola de Educação Física e Desportos (EEFD) recebe um conjunto de tendas anualmente, com conteúdos científicos e tecnológicos expostos por meio de painéis e equipamentos de multimídia; monitores e pesquisadores interagem com o público visitante, divulgando informações sobre suas áreas de conhecimento. Também são promovidas palestras de pesquisadores e cientistas convidados para o evento, bem como a distribuição de material impresso por parte dos pesquisadores expositores. O tema da SNCT de 2019 foi "Bioeconomia: diversidade e riqueza para o desenvolvimento sustentável". A FU-UFRJ desenvolveu oficinas sobre o desenvolvimento de ações sustentáveis no âmbito da manipulação de medicamentos e do URM, como estratégias para a preservação do meio ambiente, dentre as quais a ação educativa relativa a esse artigo faz parte. 
A FU-UFRJ, visando contribuir para a popularização do conhecimento, transformação social e promoção da aproximação entre os saberes produzidos na Universidade e a comunidade, participa, desde 2012, da SNCT, na UFRJ. Em cada edição, a FU oferece ao público-alvo diversas oficinas educacionais e lúdicas, ampliando a oportunidade de acesso à saúde e educação. Mendonça e Silva (2002) afirmam que poucos são os que têm acesso direto aos conhecimentos produzidos nas universidades públicas. Sendo assim, a extensão universitária é imprescindivel para a democratização do acesso a esses conhecimentos, e também para o redimensionamento da função social da própria universidade, principalmente se esta for pública. Logo, na SNCT, a FU-UFRJ tem um papel importante na difusão e popularização de conhecimentos sobre medicamentos e cuidados à saúde.

\section{RESULTADOS}

As histórias em quadrinhos elaboradas (Figura 1) foram utilizadas na SNCT entre os dias 21 e 25 de outubro de 2019, das 9 às 17 h. Os alunos da FU-UFRJ estiveram no stand da FU-UFRJ orientando o público. A dinâmica da atividade foi participativa e ativa. Esta teve como caracteristica o envolvimento de todos os participantes no processo de construção e socialização do saber (Souza, 2018). Os alunos da FU-UFRJ apresentaram a história em quadrinhos ao público, que, incentivado pela equipe da FU-UFRJ, demonstrava o nível de conhecimento que tinha sobre os conteúdos, buscando uma construção lúdica e coletiva do conhecimento.

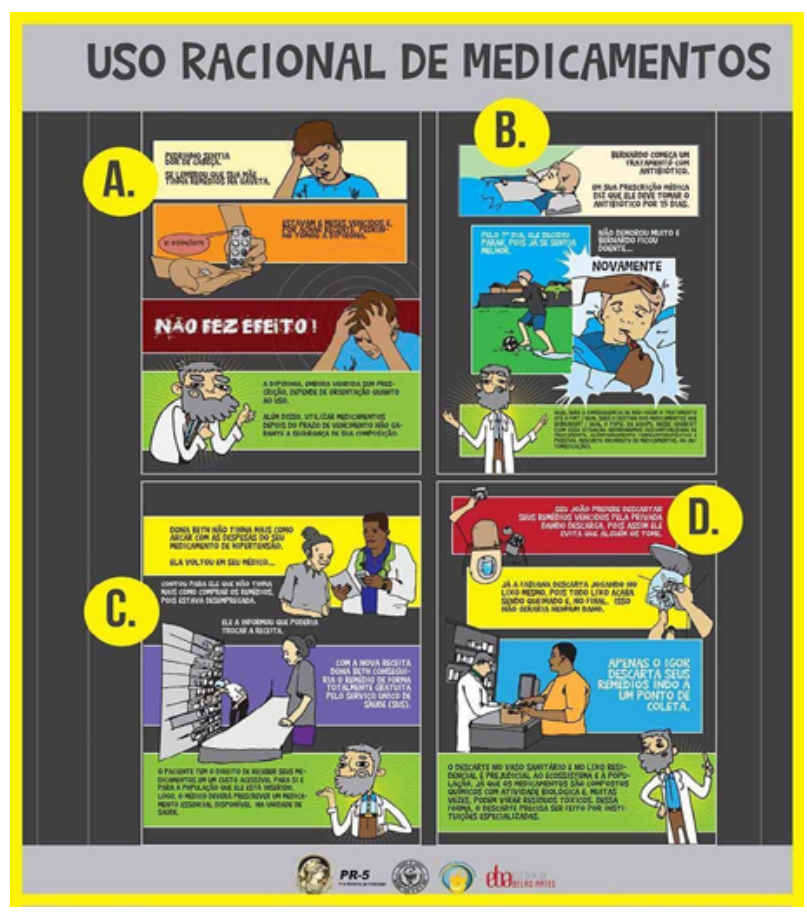


Figura 1 - Histórias em quadrinhos. A - Automedicação e uso de medicamentos vencidos; B - Descontinuidade de antimicrobianos sem acompanhamento multiprofissional; C - Descontinuidade do tratamento por questões relacionadas ao acesso; D

- Descarte e incorreto de medicamentos.

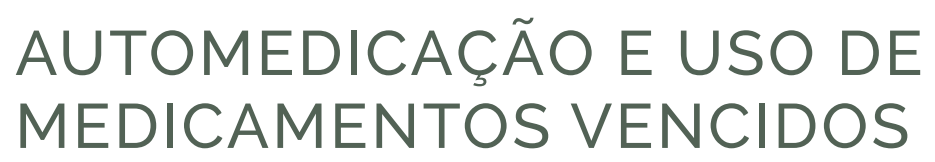

Um aspecto relevante para a promoção do uso racional de medicamentos abordado ao longo da ação foi a observação da qualidade do medicamento que está sendo administrado, estando essa relacionada à manutenção da estabilidade do fármaco frente às condições de armazenamento e manuseio, bem como a validade do mesmo (Lima et al., 2010). Nos estoques domiciliares pode haver medicamentos decorrentes de sobras de tratamentos anteriores, prescritos para tratamento de transtornos agudos e crônicos, ou por aqueles utilizados através da automedicação (Dal Pizzol et al., 2006). Isso pode gerar situações como a ausência de resposta terapêutica, que foi abordada na história em quadrinhos como mostra a Figura 1.A. Armazenar medicamentos nos domicilios é uma prática comum, e pode representar um potencial risco para o surgimento de agravos à saúde.

O armazenamento inadequado dos medicamentos pode favorecer a degradação dos princípios ativos, reduzir a sua eficácia ou promover a formação de outras substâncias tóxicas, o que também compromete a estabilidade dos medicamentos e causa risco de intoxicação (Mastroianni et al., 2011). Além disso, as pessoas costumam guardar os medicamentos fora de suas embalagens secundárias, o que dificulta a identificação da data de validade, bula, número do lote ou nome do princípio ativo. A falta dessas informações leva ao uso de medicamentos vencidos, constituindo um risco à saúde do usuário, e ao uso incorreto, pela confusão de medicamentos com características semelhantes. Dessa forma, em casos de inefetividade terapêutica, intoxicações e reações adversas, a ausência dessas informações inviabiliza a identificação do medicamento utilizado e do seu fabricante.

\section{DESCONTINUIDADE DE ANTIMICROBIANOS SEM ACOMPANHAMENTO MULTIPROFISSIONAL}

A resistência aos antimicrobianos é uma das maiores preocupações globais em saúde pública. Apesar de ocorrer naturalmente, o problema tem se acelerado e agravado a partir do uso inadequado de antimicrobianos na produ- 
ção de alimentos, no manejo de animais e nos serviços de saúde. Está cada vez mais difícil tratar um crescente número de infecções, já que os antimicrobianos usados estão se tornando não efetivos (WHO, 2015). Estima-se que no ano de 2050, caso não sejam tomadas ações efetivas para controlar os avanços da resistência aos antimicrobianos, uma pessoa morrerá a cada três segundos, o que representará 10 milhões de óbitos por ano (WHO, 2015).

O uso indiscriminado de antibióticos é registrado tanto em nível hospitalar (Souza et al., 2008) quanto na comunidade (Rauber et al., 2009). Este fato torna-se especialmente relevante, uma vez que no campo desta classe de medicamentos, as ações que são tomadas diante de um paciente podem repercutir em toda uma comunidade, principalmente no tocante à resistência bacteriana (Koch et al., 2008), podendo acarretar em danos ainda mais graves aos individuos (Spiandorello et al., 2006). Foi abordada a interrupção indevida e precoce do uso de antibióticos, descrita na Figura 1.B, já que o uso desenfreado de antibióticos sem uma cuidadosa avaliação das suas indicações apropriadas pode levar ao crescimento de cepas resistentes, ou seja, acarretar numa mutação seletiva. Dessa forma, no caso de antibióticos, o paciente deve ter o conhecimento da duração do tratamento e do intervalo entre as administrações, garantindo que haja adesão completa ao tratamento, para que não haja diminuição da concentração plasmática, ou ainda ocorra ineficácia do fármaco e surgimento de resistência bacteriana (Aronson \& Grahame Smith, 2004). Em estudo recente realizado com base nos indicadores de uso de medicamentos, da OMS, foi observado que a assistência prestada ao paciente é, muitas vezes, insuficiente, e isso pode comprometer a eficácia do tratamento (Santos \& Nitrini, 2004).

\section{DESCONTINUIDADE DO TRATAMENTO POR QUESTÕES RELACIONADAS AO ACESSO}

O acesso a medicamentos essenciais é reconhecido pela Organização das Nações Unidas como um dos cinco indicadores relacionados aos avanços na garantia do direito à saúde. O acesso aos medicamentos se dá mediante a disponibilidade destes, a capacidade aquisitiva das pessoas, sua acessibilidade geográfica e aceitabilidade, levando ao uso racional do produto (Blum et al., 2011).

Nesse contexto, os medicamentos de uso contínuo assumem grande importância no tratamento de doenças crônico-degenerativas, como a hipertensão arterial sistêmica e o diabetes mellitus, bem como no tratamento de problemas de saúde mental. Morbidades estas que apresentam prevalências crescentes no Brasil em decorrência do envelhecimento populacional. A falta de acesso a medicamentos para tratamento dessas enfermidades pode levar 
ao agravamento do quadro e aumentar os gastos com a atenção secundária e terciária (Arrais et al., 2005). Nesse sentido, a Política Nacional de Medicamentos (PNM) objetiva garantir o acesso da população aos medicamentos considerados essenciais (Pepe et al., 2010) como aborda a situação ilustrada da história em quadrinhos na Figura 1.C.

\section{DESCARTE INCORRETO DE MEDICAMENTOS}

A abordagem da história em quadrinhos voltada para "o descarte de medicamentos no vaso sanitário" (Figura 1.D) girou em torno dos impactos dos medicamentos sobre o meio ambiente. Os medicamentos são substâncias químicas ou biológicas, ditos poluentes orgânicos e contaminantes de potencial risco à saúde e ao meio ambiente, porém impedir que entrem em contato direto com a natureza é um grande desafio (Blankenstein \& Philippi Junior, 2018).

Sabe-se que, dos medicamentos consumidos, 50 a 90\% dos fármacos presentes nos produtos não são absorvidos, sendo eliminados pelas fezes e ganhando, portanto, a rede de esgoto (Hughes et al., 2013). Outro problema abordado foi o fato de que os medicamentos não utilizados, muitas vezes, são descartados no lixo comum, como o exemplo da história em quadrinhos, conferindo a esses residuos o status de "poluentes emergentes". Consequentemente, há, ao redor do mundo, fármacos residuais em águas naturais e em efluentes de estações de tratamento de esgoto (Borrely et al., 2012). Duas classes farmacológicas apresentam os maiores problemas: os antibióticos e os hormônios estrogênicos. Os antibióticos, pela sua larga utilização, são passiveis de induzir resistência microbiana. Os estrogênios, por sua vez, segundo as evidências, trazem modificações aos sistemas reprodutivos de certos organismos terrestres e aquáticos, resultando no desenvolvimento de anormalidades e deterioração reprodutiva nos organismos expostos (Bila \& Dezotti, 2003).

Todos esses tópicos foram abordados em profundidade, baseados em evidências científicas e também de forma lúdica através da história em quadrinhos.

\section{DISCUSSÃO}

Atualmente, a extensão universitária viabiliza a inserção dos acadêmicos no contato direto com a sociedade, dando-lhes oportunidade de vivenciar a aplicação dos conhecimentos da sua futura profissão, a complementaridade de outros conhecimentos, a viabilidade do aprendizado interdisciplinar e prático fora da sala de aula (Santos, 2014). Além de possibilitar novos meios e processos de produção, inovação e disponibilização de conhecimentos, permitindo a ampliação do acesso ao saber, priorizando práticas voltadas para o atendimento de necessidades sociais e estimulando a utilização das tecnologias disponíveis 
para ampliar a oferta de oportunidades e melhorar a qualidade da educação em todos os niveis (FORPROEX, 2012).

O presente trabalho demonstra a possibilidade de interação dialógica entre diferentes setores da comunidade na construção de uma estratégia educativa sobre o uso racional de medicamentos em um ambiente de popularização da ciência e com um vasto público, como na SNCT, contribuindo para a superação das desigualdades e da exclusão social. Além disso, sabe-se que outras abordagens têm sido empregadas com sucesso por outros autores e que é necessária a aplicação de metodologias que estimulem a participação e a democratização do conhecimento, como por exemplo: peças de teatro (Pereira et al., 2009), manejo e observação do objeto de estudo (Rancich et al., 1989), palestras e cartazes (Uchôa et al., 2004), entre outros.

Segundo Duro Martínez (2003), as histórias em quadrinhos exigem requisitos técnicos fundamentais para o desenvolvimento das ações em grupo. Entre elas, a adaptação da linguagem técnica científica ao nivel cultural dos integrantes dos grupos e o planejamento de um tempo de discussão após a exposição de conteúdos para que surjam as possiveis dúvidas e o profissional de saúde possa esclarecê-las. Tais requisitos foram devidamente contemplados ao longo das ações na SNCT, tornando a atividade reflexiva e como um ponto de partida para novos hábitos em relação ao URM.

De forma complementar, analisando as atividades educativas no âmbito da assistência farmacêutica, Schommer \& Cable (1996) ressaltam que a formação de profissionais farmacêuticos para a prática da Atenção Farmacêutica pode ser realizada por dois caminhos: o primeiro seria pelo treinamento, com os preceptores de estágio introduzindo novas práticas, e o segundo com os próprios acadêmicos, por meio de simulações de atendimento com foco no aprendizado do paciente. Nessas simulações os alunos devem ser ensinados a ter uma postura ativa no desenvolvimento da relação com os pacientes. Tal relação foi profundamente significativa para os estudantes da FU-UFRJ que participaram ativamente das atividades, como mostra a Figura 2.A.

Figura 2: A - Aluna da FU-UFRJ apresentando as histórias em quadrinhos desenvolvidas para a apresentação na SNCT. B - Alunos de escolas públicas do Rio de Janeiro participando ativamente das histórias.
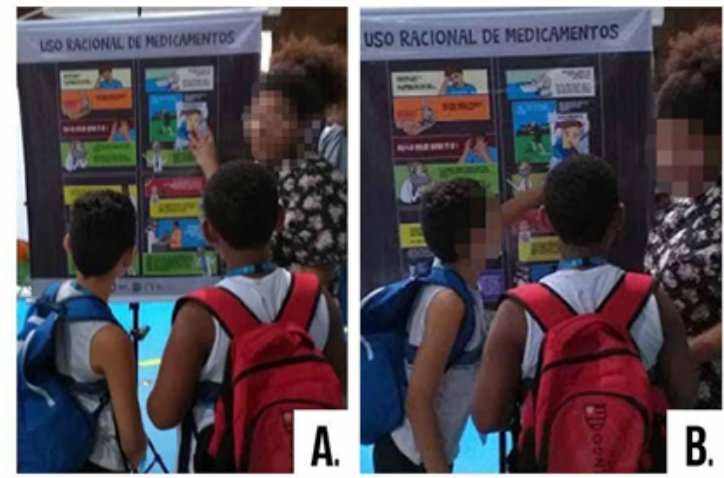
As atividades desenvolvidas na SNCT constituíram aportes decisivos para a ampliação do universo de referência e para inserção do aluno de graduação em questões contemporâneas. Dessa maneira, o enriquecimento da experiência discente pôde ser proporcionado pela conexão do conhecimento teórico aos desafios da realidade (FORPROEX, 2012). Além disso, os alunos foram conscientizados de sua função como facilitadores na divulgação do conhecimento e na aplicação deste na vida cotidiana das pessoas em sociedade. Os estudos vêm demonstrando o impacto positivo das ações de saúde para a inserção desses futuros profissionais de saúde nas equipes ligadas ao Sistema Único de Saúde (SUS) (Kotecki et al., 2000). Sabe-se que já está bem evidenciado o problema do uso irracional de medicamentos, e que uma das formas de reverter esse processo é pela educação e informação da população (Pfaffenbach et al., 2002). As campanhas educativas, portanto, são altamente recomendadas para esse processo de conscientização (Rauber et al., 2009). O ensino sobre URM é fundamental para a autonomia do individuo em seu autocuidado e para a divulgação do conhecimento adquirido nas ações.

No contexto de campanhas educativas, a SNCT é um importante ambiente de popularização do conhecimento científico. A semana conta com a participação ativa do público externo à universidade, como mostra a figura 2.B. Segundo Mancuso (2000), a realização de feiras de ciências traz benefícios para toda a comunidade. O autor destaca as seguintes mudanças nos participantes de ações educativas: (1) crescimento pessoal e ampliação dos conhecimentos; (2) ampliação da capacidade comunicativa; devido à troca de ideias; (3) mudanças de hábitos e atitudes com o desenvolvimento da autoconfiança e da iniciativa em construir o conhecimento; (4) desenvolvimento da criticidade devido ao amadurecimento de avaliação das informações; (5) maior envolvimento e interesse em pesquisas; (6) exercício da criatividade; e (7) maior politização dos participantes, devido à ampliação da visão de mundo. Dessa maneira, a participação em feiras de ciências representa a culminação de um processo de estudo, investigação e produção que tem por objetivo a formação científica dos estudantes.

A experiência interdisciplinar desenvolvida durante a SNCT, por intermédio de discussão, ação, reflexão e transformação, contribuiu ativamente para estimular o espírito crítico, a reflexão sobre a cidadania e o papel social da educação superior, confirmando a indissociabilidade da relação ensino-pesquisa-extensão. Sob essa perspectiva, os estudantes foram protagonistas da sua formação técnica, visto que participaram de forma ativa na obtenção das competências necessárias à atuação no projeto: contribuiram com ideias no processo de construção do saber, unindo a teoria com a prática; desenvolveram atividades em equipe; e, ainda, aprenderam a investigar as fontes de informação antes de disseminar um conteúdo. Os acadêmicos também foram protagonistas da sua formação cidadã, pois se reconheceram como agentes de transformação social quando em contato com as questões e demandas da sociedade, e entenderam a necessidade de respeitar opiniões diferentes (FORPROEX, 2012). 
A abordagem do tema, somada ao ambiente da SNCT, foi uma estratégia interessante para a divulgação do conhecimento desenvolvido pela equipe de FU-UFRJ. A utilização de história em quadrinhos, de acordo com todo o exposto, pode ser um instrumento útil de educação em saúde. Além disso, a participação na SNCT consolidou a ação cidadã por parte da Universidade, com a efetiva difusão e democratização dos saberes nela produzidos. Essa atuou junto ao sistema de ensino público, fortalecendo a educação básica através da contribuição técnico-científica.

\section{CONSIDERAÇÕES FINAIS}

Por meio desta experiência foi possivel desenvolver uma estratégia de educação em saúde na SNCT 2019, lançando mão de história em quadrinhos. A utilidade desse gênero para o conhecimento do público foi constatada, uma vez que as histórias retrataram pontos levantados pela literatura sobre o uso racional de medicamentos. Elas também atraíram de maneira considerável o público que participou do evento.

Foi possivel desenvolver um diálogo entre artes e ciências da saúde, respeitando as habilidades singulares de ambos os conhecimentos e desenvolvendo instrumentos de divulgação do conhecimento científico para além do contexto universitário.

A partir destas considerações, percebe-se o estímulo às ações multidisciplinares desenvolvidas por profissionais de saúde e da área acadêmica junto ao público extramuros da universidade como forma de implantar a educação em saúde sobre diferentes temas. Além disso, sugere-se que as ações, sempre que possivel, sejam pautadas no conhecimento mútuo, em uma visão multidisciplinar e baseada em evidências cientíicas. Foi possivel aferir o resultado positivo da ação na SNCT pela participação dos alunos em toda a campanha, pelo interesse continuado e dúvidas após o momento do compartilhamento da história em quadrinhos. 


\section{REFERÊNCIAS}

Alcântara, C. S. de, \& Bezerra, J. A. B. (2016). O lúdico, a escola e a saúde: a educação alimentar no gibi. Trabalho, Educação e Saúde, v. 14(3), p. 889-904. Recuperado de https://doi.org/10.1590/1981-7746-solooozo. Acesso em 23 Jun. 2020.

Aronson, J. K., \& Grahame Smith, D. (2004). Tratado de farmacologia clínica e farmacoterapia. Editora Guanabara Koogan.

Arrais, P. S. D., Coelho, H. L. L., Batista, M. D. C. D. S., Carvalho, M. L., Righi, R. E., \& Arnau, J. M. (1997). Perfil da automedicação no Brasil. Revista de Saúde Publica, v. 31(1), p. 71-77. Recuperado de https://doi.org/10.1590/s003489101997000100010. Acesso em 23 Jun. 2020.

Arrais, P. S. D., Brito, L. L., Barreto, M. L., \& Coelho, H. L. L. (2005). Prevalence and determinants of medicines consumption in Fortaleza, Ceará, Brazil. Cadernos de Saúde Pública, v. 21(6), p. 1737-1746. Recuperado de https://doi.org/10.1590/ S0102-311X2005000600021. Acesso em 23 Jun. 2020.

Barros, J. A. C. (2004). Políticas farmacêuticas: a serviço dos interesses da saúde? Editora Unesco. Recuperado de http://www6.ensp.fiocruz.br/visa/?q=node/4841. Acesso em 23 Jun. 2020.

Bila, D. M., \& Dezotti, M. (2003). Pharmaceutical drugs in the environment. Quimica Nova, v. 26(4), p. 523-530. Recuperado de https://doi.org/10.1590/s010040422003000400015. Acesso em 23 Jun. 2020.

Blankenstein, G. M. P. Philippi, J. A. (2018). O descarte de medicamentos e a política nacional de residuos sólidos: uma motivação para revisão das normas sanitárias. R. Dir. sanit., v. 19(1), p. 50-74. Recuperado de http://www.revistas.usp. $\mathrm{br} / \mathrm{rdisan} / \mathrm{article/view/148124.} \mathrm{Acesso} \mathrm{em} 23$ Jun. 2020.

Blum, B., Luiza, V. L., \& Bermudez, J. A. Z. (2011). Acesso a medicamentos medido no nivel domiciliar: a proposta da OMS e outras iniciativas brasileiras. Rev Bras Farm, v. 92(3), p. 223-31.

Bonfim, M. A. A. (2015) A visão de alunos do ensino fundamental sobre ciência e tecnologia: Um estudo de caso sobre a contribuição da semana nacional de C\&T. Dissertação para obtenção do título de Mestre em Ciência, Tecnologia e Educação, do Centro Federal de Educação Tecnológica Celso Suckow da Fonseca. Rio de Janeiro. 
Borrely, S. I., Caminada, S. M. L., Ponezi, A. N., Santos, D. R. dos, \& Silva, V. H. O. (2012). Contaminação das águas por residuos de medicamentos: Ênfase ao cloridrato de fluoxetina. Mundo Da Saúde, p. 556-563. Recuperado de https:// repositorio.unesp.br/handle/11449/73640. Acesso em 23 Jun. 2020.

Brasil. Ministério da Saúde (2012). Secretaria de Ciência, Tecnologia e Insumos Estratégicos. Uso racional de medicamentos: temas selecionados / Ministério da Saúde, Secretaria de Ciência, Tecnologia e Insumos Estratégicos. Brasília: Ministério da Saúde. Recuperado de https://saude.gov.br/saude-de-a-z/uso -racional-de-medicamentos. Acesso em 23 Jun. 2020.

Caruso, F., De Carvalho, M., \& Silveira, M. C. (2002). Uma proposta de ensino e divulgação de ciências através dos quadrinhos. Ciência \& Sociedade, v.8, p.1-9. Recuperado de https://d1wqtxts1xzle7.cloudfront.net/35659001/quadrinhos_ em_ciencias.pdf. Acesso em 23 Jun. 2020.

Dal Pizzol, T. da S., Piccoli, A., Brugnera, Q., Schenkel, E. P., \& Mengue, S. S. (2006). Essential medicines stored in households in Southern Brazil. Acta Farm. Bonaerense, v. 25, p.601-607. Recuperado de https://www.researchgate.net/publication/291758110. Acesso em 23 Jun. 2020.

Darnaud, R., \& Dato, M. I. (2009). Educación para la salud en escuelas argentinas: Concurso de plástica como actividad motivadora. Revista Panamericana de Salud Publica/Pan American Journal of Public Health, v. 25(2), p. 181-187. Recuperado de https://doi.org/10.1590/s1020-49892009000200013. Acesso em 23 Jun. 2020.

Ducati, L. A., Fonseca M. B., Franca De Araújo, G., Zini, U. L., Gonçalves, R. M. R., \& Andreoli, P. T. D. J. (2005). Monitoração de propaganda e publicidade de medicamentos: mbito de São Paulo. Brazilian Journal of Pharmaceutical Sciences, v. 41(3), p. 345-349. Recuperado de https://doi.org/10.1590/s151693322005000300007. Acesso em 23 Jun. 2020.

Duro Martínez, J. C. (2003). El Discurso de los Profesionales de Atención Primaria de la Comunidad de Madrid Acerca del Trabajo con Grupos: Sobre Técnicas y Técnicos. Revista Espanola de Salud Publica, v. 77(5), p. 615-627. Recuperado de https://doi.org/10.1590/s1135-57272003000500010. Acesso em 23 Jun. 2020.

Elisa, M., Carbonari, E., \& Pereira, A. C. (2015). A extensão universitária no Brasil, do assistencialismo à sustentabilidade. Revista de Educação, v. 10, p. 1-6. Recuperado de https://pdfs.semanticscholar.org/d7cd/ob5a59d228e8b6ec031a57350211b5e213d1.pdf. Acesso em 23 Jun. 2020.

Fórum de Pró-reitores de Extensão das instituições públicas deeducação superior brasileira (FORPROEX). Política Nacional de Extensão Universitária. Porto 
Alegre: UFRGS/Pró-Reitoria de Extensão, 2012.

Hughes, S. R., Kay, P., \& Brown, L. E. (2013). Global synthesis and critical evaluation of pharmaceutical data sets collected from river systems. American Chemical Society, v. 47, p. 661-667. Recuperado de . https://doi.org/10.1021/es3030148. Acesso em 23 Jun. 2020.

Koch, C. R., Ribeiro, J. C., Schnor, O. H., Zimmermann, B. S., Müller, F. M., D'Agostin, J., Machado, V., \& Zhang, L. (2008). Resistência antimicrobiana dos uropatógenos em pacientes ambulatoriais, 2000-2004. Revista Da Sociedade Brasileira de Medicina Tropical, v. 41(3), p. 277-281. Recuperado de https://doi.org/10.1590/ S0037-86822008000300010. Acesso em 23 Jun. 2020.

Kotecki, J. E., Elanjian, S. I., \& Torabi, M. R. (2000). Health promotion beliefs and practices among pharmacists. Journal of the American Pharmaceutical Association, v. 40(6), p. 773-779. Recuperado de https://doi.org/10.1016/ S1086-5802(16)31124-X. Acesso em 23 Jun. 2020.

Lima, G. B., Nunes, L. C. C., \& de Barros, J. A. C. (2010). Uso de medicamentos armazenados em domicilio em uma população atendida pelo Programa Saúde da Familia. Ciência e Saúde Coletiva, 15(SUPPL. 3), 3517-3522. Recuperado de https://doi.org/10.1590/s1413-81232010000900026.

Luiza, V. L., Mendes, L. V. P., Tavares, N. U. L., Bertoldi, A. D., Fontanella, A. T., Oliveira, M. A., Campos, M. R., Arrais, P. S. D., .. Farias, M. R. (2019). Inappropriate use of medicines and associated factors in Brazil: an approach from a national household survey. Health Policy and Planning, v. 34, p.27-35. Recuperado de https://doi.org/10.1093/HEAPOL. Acesso em 23 Jun. 2020.

Mancuso, R. (2000). Feiras de Ciências: produção estudantil, avaliação, conseqüências. Contexto Educativo: Revista Digital de Investigación y Nuevas Tecnologías, v. 6, p. 8. Recuperado de https://dialnet.unirioja.es/servlet/articulo? codigo=1024170. Acesso em 23 Jun. 2020.

Mastroianni, P. de C., Lucchetta, R. C., Sarra, J. dos R., \& Galduróz, J. C. F. (2011). Estoque doméstico e uso de medicamentos em uma população cadastrada na estratégia saúde da familia no Brasil 1. Rev. Panam. Salud Publica, v. 29(5), p. 358-364. Recuperado de https://www.scielosp.org/pdf/rpsp/2011.v29n5/358364. Acesso em 23 Jun. 2020

Matos, G. C., Rozenfeld, S., \& Bortoletto, M. E. (2002). Intoxicações medicamentosas em crianças menores de cinco anos. Revista Brasileira de Saude Materno Infantil, v. 2(2), p.167-176. Recuperado de https://doi.org/10.1590/ s1519-38292002000200009. Acesso em 23 Jun. 2020. 
Mendonça, S. G. L, Silva, P. S. A. (2002). Extensão universitária: uma nova relação com a administração pública. In Calderón, A. I. \& Sampaio, H. (orgs). Extensão Universitária: ação comunitária em universidades brasileiras. São Paulo, v. 3, p. 29-44.

Minayo, M. D. S. (2008). O desafio do conhecimento. Pesquisa qualitativa em saúde. Editora Hucitec, $9^{\text {a }}$ Edição.

Morato, M. A., Struchiner, M., Bordoni, E., \& Ricciardi, R. M. V. (1998). Representação visual de estruturas biológicas em materiais de ensino. História, Ciências, Saúde-Manguinhos., v. 5(2),p. 415-433. Recuperado de https://doi.org/10.1590/ s0104-59701998000200007. Acesso em 23 Jun. 2020.

Negrete, A., \& Lartigue, C. (2004). Learning from education to communicate science as a good story. Endeavour, v. 28, p.120-124. Recuperado de https://doi. org/10.1016/j.endeavour.2004.07.003. Acesso em 23 Jun. 2020.

Negrete, A. (2019). Science via fictional narratives. Ludus Vitalis, v. 10, p. 197-204. Disponivel em: http://www.ludus-vitalis.org/ojs/index.php/ludus/article/ viewFile/584/586. Acesso em 23 Jun. 2020.

Oliveira, T. B. M., \& Presoto, L. H. (2009). Effectiveness of a health promotion in pre-school infants program in the city of Anápolis, Goiás State. Ciência \& Saúde Coletiva, v. 14(5), p. 1891-1902. Recuperado de https://doi.org/10.1590/S141381232009000500031 . Acesso em 23 Jun. 2020.

Organização Pan Americana de Saúde (2007). Relatório de Oficina de trabalho. Uso racional de medicamentos na perspectiva multiprofissional. Brasilia. Recuperado de https://www.paho.org/bra/index.php?option=com_docman\&view=list\&ltemid=965\&slug=medicamentos-tecnologia-e-pesquisa-075. Acesso em 23 Jun. 2020.

Pepe, V. L. E., Ventura, M., Sant'ana, J. M. B., Figueiredo, T. A., de Souza, V. dos R., Simas, L., \& Osorio de Castro, C. G. S. (2010). Caracterização de demandas judiciais de fornecimento de medicamentos "essenciais" no Estado do Rio de Janeiro, Brasil. Cadernos de Saude Publica, v. 26(3), p. 461-471. Recuperado de https://doi.org/10.1590/s0102-311X2010000300004. Acesso em 23 Jun. 2020.

Pereira, B., Silva, K. S. S., \& Souza, R. P. de. (2009). Um cidadão não nasce grandão. Saúde e Sociedade, v. 18, p. 93-95. Recuperado de https://repositorio.usp.br/ bitstream/handle/BDPI/6364/art_PEREIRA_Um_cidadao_nao_nasce_grandao_2009.pdf?sequence=1. Acesso em 23 Jun. 2020.

Pfaffenbach, G., Carvalho, O. M., \& Bergsten-Mendes, G. (2002). Drug adverse reactions leading to hospital admission. Revista Da Associação Médica 
Brasileira, v. 48(3), p. 237-241. Recuperado de https://doi.org/10.1590/So10442302002000300037. Acesso em 23 Jun. 2020.

Rama, A., \& Vergueiro, W.. (2008). Como usar as histórias em quadrinhos na sala de aula. Editora Contexto.

Rancich, A. M., Ferrarini, S. O., \& Homero Palma, N. (1989). Experiencia de enseñanza-aprendizaje: cambio de creencias y conductas en el manejo del agua de consumo. Revista de Saude Publica, v. 23(3), p. 183-188. Recuperado de https://doi.org/10.1590/s0034-89101989000300002. Acesso em 23 Jun. 2020.

Rauber, C., Feltrin, M. R., \& Piovezan, A. P. (2009). Evaluation of antibiotics dispensing profile in Tubarão, Santa Catarina, Brazil. Brazilian Journal of Pharmaceutical Sciences, v. 45(4), p.787-793. Recuperado de https://doi.org/10.1590/ S1984-82502009000400024. Acesso em 23 Jun. 2020.

Rebolho, M. C. T., Casarotto, R. A., \& João, S. M. A. (2009). Estratégias para ensino de hábitos posturais em crianças: história em quadrinhos versus experiência prática. Fisioterapia e Pesquisa, v. 16(1), p. 46-51. Recuperado de https://doi. org/10.1590/s1809-29502009000100009. Acesso em 23 Jun. 2020.

Rodrigues, A. L. L., Costa, C. L. N. do A., Prata, M. S., Batalha, T. B. S., \& Neto, I. de F. P. (2013). Contribuições da extensão universitária na sociedade. Caderno de Graduação - Ciências Humanas e Sociais - UNIT - SERGIPE, v. 1(2), p. 141-148. Recuperado de https://periodicos.set.edu.br/index.php/cadernohumanas/ article/view/494. Acesso em 23 Jun. 2020.

Santos, V., \& Ottati Oliveira Nitrini, S. M. (2004). Indicadores do uso de medicamentos prescritos e de assistência ao paciente de serviços de saúde. Revista de Saúde Pública, v. 38(6), p. 819-826. Recuperado de https://doi.org/10.1590/ s0034-89102004000600010. Acesso em 23 Jun. 2020.

Santos, M. P. (2014). A extensão universitária como "laboratório" de ensino, pesquisa científica e aprendizagem profissional: um estudo de caso com estudantes do curso de licenciatura em pedagogia de uma faculdade particular do estado do Paraná. Extensio: Revista Eletrônica de Extensão, v. 11(18), p. 36-52. Recuperado de https://dialnet.unirioja.es/servlet/articulo?codigo=6184534. Acesso em 23 Jun. 2020.

Schommer, J. C., \& Cable, G. L. (1996). Current status of pharmaceutical care practice: Strategies for education. American Journal of Pharmaceutical Education, v. 60(1), p. 36-41. Recuperado de https://experts.umn.edu/en/publications/current-status-of-pharmaceutical-care-practice-strategies-for-edu. Acesso em 23 Jun. 2020. 
Souza, A. L. L. (2000). A história da extensão universitária. 1.ed. Campinas: Editora Alínea.

Souza, H. P., Vilhordo, D. W., Breigeiron, R., Alessandretti, M. B., \& Silva, T. G. B. (2008). Auditoria no uso de antimicrobianos em enfermaria cirúrgica. Revista do Colegio Brasileiro de Cirurgiões, v. 35(4), p. 216-220. Recuperado de https://doi. org/10.1590/s0100-69912008000400002. Acesso em 23 Jun. 2020.

Souza, J. C. (2018). Promoção da Saúde e Segurança dos Alimentos, uma Abordagem em Saúde Coletiva. Revista Extensão. XIV, n. 1.

Spiandorello, W. P., Faintuch, J., Ribeiro, G. T., Karkow, F. J., \& Alvares, J. O. (2006). Use of multiple antimicrobial drugs by clinical patients: A prognostic index of hospital mortality? Clinics, v. 61(1), p. 15-20. Recuperado de https://doi. org/10.1590/S1807-59322006000100004. Acesso em 23 Jun. 2020.

Uchôa, C. M. A., Serra, C. M. B., Magalhães, C. de M., Silva, R. M. M. da, Figliuolo, L. P., Leal, C. A., \& Madeira, M. de F. (2004). Health education: teaching about American tegumentary leishmaniasis. Cadernos de Saúde Pública, v. 20(4), p. 935-941. Recuperado de https://doi.org/10.1590/S0102-311X2004000400007. Acesso em 23 Jun. 2020.

Universidade Federal do Rio de Janeiro. (2004). Semana Nacional de Ciência e Tecnologia. Recuperado de https://snct.pr5.ufrj.br/. Acesso em 23 Jun. 2020.

WHO - World Health Organization. (1985). Sudden cardiac death: report of a WHO scientific group [meeting held in Geneva from 24 to 27 October 1984]. Recuperado de https://apps.who.int/iris/handle/10665/39554. Acesso em 16 Jun. 2020.

WHO - World Health Organization. (2015). Worldwide country situation analysis: response to antimicrobial resistance: summary (No. WHO/HSE/PED/AIP/2015.1). World Health Organization. Recuperado de https://apps.who.int/iris/bitstream/handle/10665/163473/WHO_HSE_PED_AIP_2015.1_eng.pdf. Acesso em 16 Jun. 2020.

Data de submissão: 28/07/2020

Data de aceite: $20 / 10 / 2020$ 